\title{
The long-term evolution of the X-ray pulsar XTE J1814-338: A receding jet contribution to the quiescent optical emission? ${ }^{\star}$
}

\author{
M. C. Baglio ${ }^{1,2}$, P. D’Avanzo ${ }^{1}$, T. Muñoz-Darias ${ }^{3}$, R. P. Breton ${ }^{3}$, and S. Campana ${ }^{1}$
}

\author{
1 INAF, Osservatorio Astronomico di Brera, via E. Bianchi 46, 23807 Merate (Lc), Italy \\ e-mail: cristina.baglio@brera.inaf.it \\ 2 Università degli Studi di Milano, Dipartimento di Fisica, via Celoria 16, 20133 Milano, Italy \\ 3 School of Physics and Astronomy, University of Southampton, SO17 1BJ, UK
}

Received 3 August 2013 / Accepted 23 September 2013

\begin{abstract}
Aims. We present a study of the quiescent optical counterpart of the accreting millisecond X-ray pulsar XTE J1814-338 that is aimed at unveiling the different components, which contribute to the quiescent optical emission of the system.

Methods. We carried out multiband $(B V R)$ orbital phase-resolved photometry of the system using the ESO Very Large Telescope (VLT) that is equipped with the FORS2 camera, covering about $70 \%$ of the 4.3 hour orbital period.

Results. The optical light curves are consistent with a sinusoidal variability that are modulated with an orbital period with a semiamplitude of 0.5-0.7 mag. They show evidence of a strongly irradiated companion star, which agrees with previous findings for this system. However, the observed colours cannot be accounted for by the companion star alone, suggesting the presence of an accretion disc during quiescence. The system seems to be fainter in all analysed bands compared to previous observations. The $R$ band light curve displays a possible phase offset with respect to the $B$ and $V$ band. Through a combined fit of the multi-band light curve performed with a Markov chain Monte Carlo technique, we derive constraints on the companion star, disc fluxes, system distance, and companion star mass.

Conclusions. The irradiation luminosity required to account for the observed day-side temperature of the companion star is consistent with the spin-down luminosity of a millisecond radio pulsar. Compared to our data with previous observations, which were collected over 5 years, the flux decrease and spectral evolution of the observed quiescent optical emission cannot be satisfactorily explained with the combined contribution of an irradiated companion star and of an accretion disc alone. The observed progressive flux decrease as the system gets bluer could be due to a continuum component that evolves towards a lower, bluer spectrum. While most of the continuum component is likely due to the disc, we do not expect it to become bluer in quiescence. Hence, we hypothesize that an additional component, such as synchrotron emission from a jet was significantly contributing in the data obtained earlier during quiescence and then progressively fading or moving its break frequency towards longer wavelengths.
\end{abstract}

Key words. X-rays: binaries - stars: neutron - stars: jets

\section{Introduction}

Fifteen accreting millisecond X-ray pulsars (AMXPs) have been discovered so far. These transient systems are a subclass of low mass X-ray binaries (LMXBs), that host a weakly magnetic neutron star which has been spun up to millisecond periods by angular momentum transfer from a donor companion of mass $M \lesssim 1 M_{\odot}$. These systems have an orbital period in the range of $40 \mathrm{~min}$ to $19 \mathrm{hr}$ and spin frequencies between 1.7 and $5.4 \mathrm{~ms}$ (see Patruno \& Watts 2012 for a recent review).

These systems are suspected to be the missing link between LMXBs and millisecond radio pulsars, the former being the progenitors of the latter. According to the standard model for the formation of millisecond radio pulsars, these objects come from the recycling of neutron stars hosted in LMXBs, which have been spun up by the accretion torques that result from the transfer of mass with a large angular momentum from the companion star. The radio emission switches off during the active mass transfer phase and resumes again when it comes to an end, leaving behind a recycled radio pulsar, which looses rotational

* Based on observations made with ESO Telescopes at the Paranal Observatory under programme ID 383.D-0730(A). energy in the form of a relativistic particle wind that can irradiate the companion star.

The first confirmation of this scenario has come with the observation of PSR J1023+0038, also known as missing link pulsar (Archibald et al. 2009), which shows that LMXBs can turn on as radio millisecond pulsars. Furthermore, the recent detection of transient millisecond X-ray pulsations from the system IGR J18245-2452, which was known to be a millisecond radio pulsar, provided the direct observational evidence of the recycling scenario (Papitto et al. 2013).

Optical observations of AMXPs in quiescence offer the only chance to study the characteristics of the companion star, which would be otherwise veiled by the bright disc emission during active phases. In LMXBs, which comprise a companion star suffering significant tidal distortion, the light curves in quiescence are characterised by orbital variability due to ellipsoidal modulation. This effect arises from the observation that the projected surface area of the distorted star is larger at quadratures than at conjunctions, hence resulting in maxima and minima of the total flux at those two sets of phases, respectively. Gravity darkening introduces a slight asymmetry in the double modulation, making the minimum at the superior conjunction of the companion deeper because of the lower surface gravity. However, the 
companion star in systems with short orbital periods (and hence with a smaller orbital separation) might be severely irradiated by the compact object and/or by the residual accretion disc. In such cases, the light curve would be dominated by the contribution from the irradiation effect, which displays a maximum at the superior conjunction of the companion and a minimum at the inferior conjunction. Additional features can be observed in the light curve profiles, particularly if a residual accretion disc significantly contributes to the overall optical emission during quiescence (see, e.g., Hynes 2010). Detailed multi-band optical and near-infrared orbital-phase resolved monitoring of these systems can provide useful information in disentangling the different components contributing to their total quiescent emission.

The binary system XTE J1814-338 was discovered during an outburst on 2003 June 5 (MJD 52795), by the Rossi X-ray Timing Explorer (RXTE; Markwardt \& Swank 2003). The system is characterised by coherent pulsations at a frequency of $314.3 \mathrm{~Hz}(3.18 \mathrm{~ms})$ with an orbital modulation of $4.3 \mathrm{~h}$. Thanks to the observation of type I X-ray bursts, Strohmayer $\&$ Watts (2005) estimated the distance of this system to be $8.0 \pm$ $1.6 \mathrm{kpc}$. During the outburst, an $R \sim 18.3$ optical counterpart was identified, and hydrogen and helium emission lines were detected through optical spectroscopy, suggesting for a nondegenerate companion (Krauss et al. 2005). The quiescent optical counterpart observed in 2004 is faint $(R \sim 22.5$ and $V \sim 23.3$; D'Avanzo et al. 2009). The orbital phase-resolved quiescent optical light curves display a sinusoidal profile showing a maximum at the superior conjunction of the companion. This behaviour suggests that irradiation from the compact object plays a key role at heating the inner face of the companion star in the observed optical quiescent luminosity. This has been intepreted as the indirect signature of an energetic rotation-powered recycled millisecond pulsar (D'Avanzo et al. 2009 and references therein).

In this paper, we present ESO-VLT orbital phase-resolved multi-band optical imaging observations of XTE J1814-338 that are obtained on September 2009 with archival data obtained in May 2004. These observations are complementary to those presented in D'Avanzo et al. (2009). These datasets were obtained while the source was in quiescence and covered a period of five years. No outburst occurred between the two epochs.

The paper is organized as follows: in Sect. 2 we present our data. In Sect. 3 we compare our results with the findings of D'Avanzo et al. (2009) and present a discussion on the origin of the quiescent optical emission of XTE J1814-338. Our conclusions are reported in Sects. 4 and 5. Through the paper, all errors are at $68 \%$ confidence level unless stated differently.

\section{Observations and data analysis}

The system XTE J1814-338 was observed in quiescence on 10 September 2009 with the ESO Very Large Telescope (VLT), using the FORS2 camera in imaging mode with the $B, V, R$ filters. The night was clear with seeing in the range $0.6^{\prime \prime}-2.5^{\prime \prime}$, which degraded through the night. A set of 29 images with 3 minutes of integration each was obtained for each filter, covering more than one orbital period. However, only images obtained under seeing $\lesssim 1^{\prime \prime}$ had a quality good enough for our purposes, due to the crowdness of the field. We thus retain 19 images for the $B$ and $V$ filters and 16 for the $R$ one, which cover about $70 \%$ of the system's orbital period.

From the ESO Archive we also retrieved $I$ band FORS2 VLT imaging data that were obtained on 2004 May 21 and 22, which complement the $R$ and $V$ data reported in D'Avanzo et al. (2009).

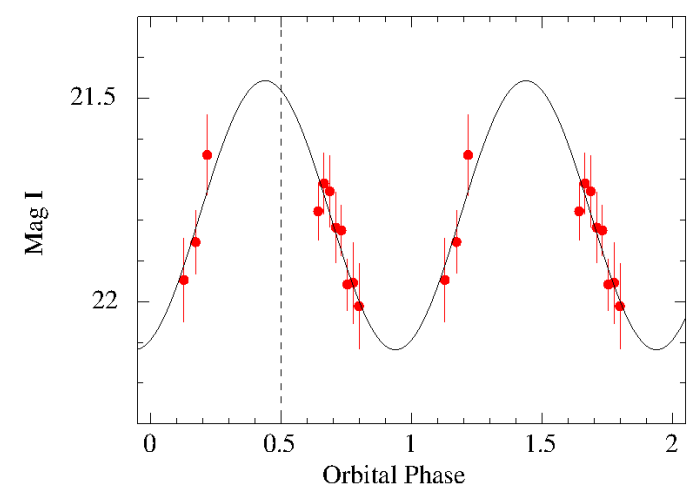

Fig. 1. I band light curve for the system XTE J1814-338 for the 2004 observation. Phase 0.0 corresponds to the inferior conjunction of the companion. We calculated the phases based on the X-rays ephemeridis of Papitto et al. (2007). Two periods of the system are drawn for clarity.

Image reduction was carried out following standard procedures: subtraction of an averaged bias frame and division by a normalised flat frame. Astrometry was performed using the USNOB $1.0^{1}$ catalogue. Point spread function-photometry was made with the ESO-MIDAS ${ }^{2}$ daophot $^{3}$ task for all the objects in the field. The photometric calibration was done against the Stetson standard field star PG2213 (Stetson 2000). To minimize any systematic effect, we performed differential photometry with respect to a selection of eight local isolated and non-saturated reference stars, which were also used as secondary standards to cross-check the calibration of the 2004 and 2009 data in the $V$ and $R$ band.

\section{Results}

\subsection{I band data}

The 2004 I band light curve shows variability consistent with a sinusoid that is modulated at the $4.3 \mathrm{~h}$ orbital period with a semi-amplitude of $0.33 \mathrm{mag}$ and an average magnitude of $I=21.79 \pm 0.04$ (Fig. 1; Table 1). The light curve shows a single minimum around phase 0.0 (inferior conjunction of the companion) and a maximum around 0.5 (superior conjunction of the companion), which are generally consistent with the $V$ and $R$ light curves obtained at the same epoch and reported in D’Avanzo et al. (2009) (see Table 1).

\subsection{9 optical light curves}

The optical counterpart of XTE J1814-338 (Krauss et al. 2005; D'Avanzo et al. 2009) is well detected in all our $B, V$, and $R$ frames obtained under seeing $\lesssim 1^{\prime \prime}$ (see Sect. 2). The optical phase resolved $B V R$ light curves are shown in Fig. 2. The source in 2009 displays a clear sinusoidal variability, modulated at the $4.3 \mathrm{~h}$ orbital period with a $0.5-0.7 \mathrm{mag}$ semi-amplitude (Table 1). The $B$ and $V$ band light curves show a single minimum around phase 0.0 and a maximum consistent with phase 0.5 (based on the precise X-ray ephemerides by Papitto et al. 2007; see Table 1).

The same behaviour during quiescence was observed on May 2004 (D'Avanzo et al. 2009), suggesting that the compact object is heating the companion star. Such orbital modulated

\footnotetext{
http://www.nofs.navy.mil/data/fchpix/

2 http://www.eso.org/projects/esomidas/

3 http://http://www.star.bris.ac.uk/ mbt/daophot/
} 

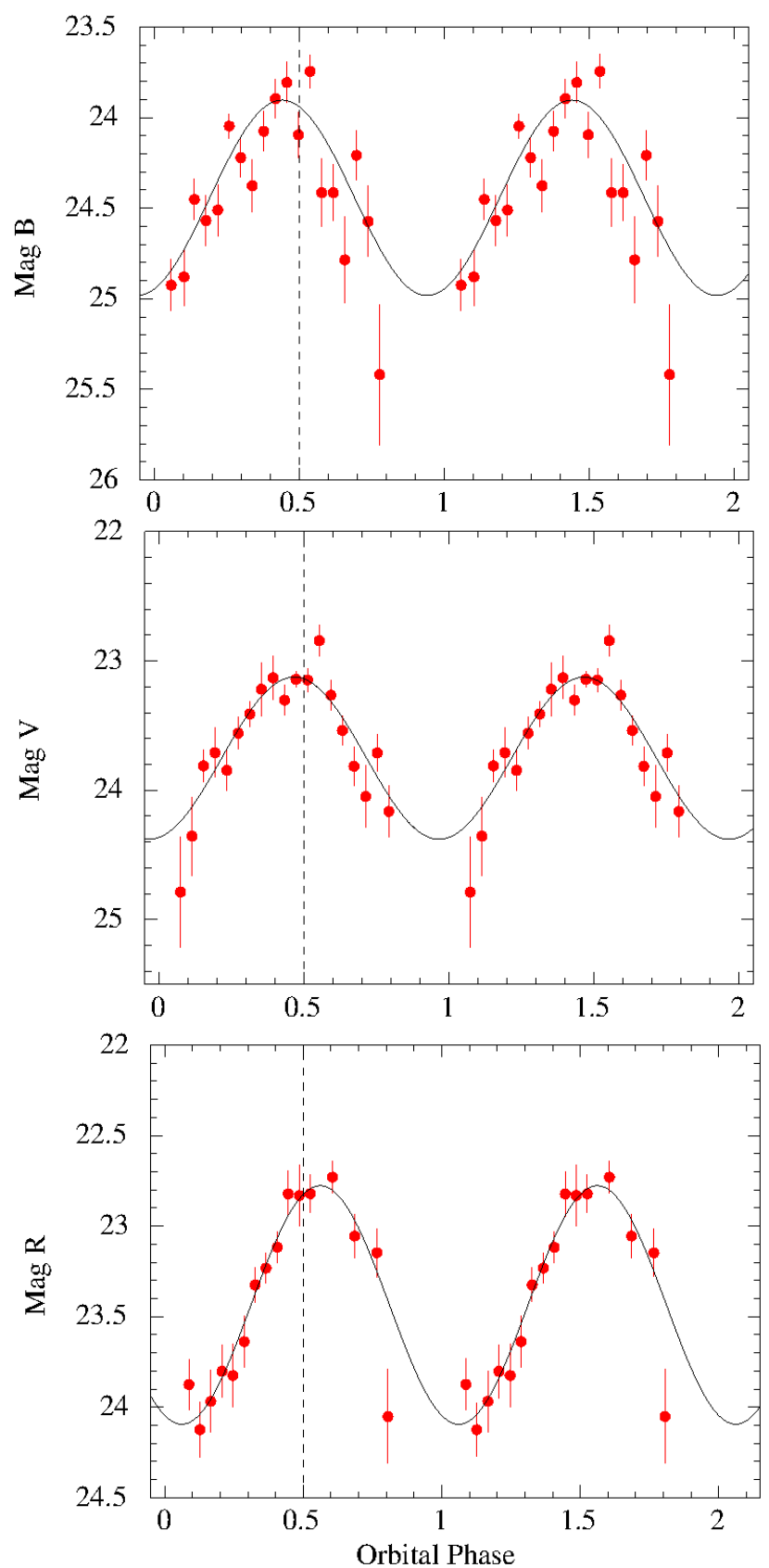

Fig. 2. $B, V, R$ light curves for the system XTE J1814-338 for the 2009 observation. Phase 0 corresponds to the inferior conjunction of the companion. We calculated the phases based on the X-rays ephemeridis of Papitto et al. (2007). Two periods of the system are drawn for clarity.

variability is also reminiscent of the quiescent optical light curves of other AMXPs (Homer et al. 2001; Campana et al. 2004; D'Avanzo et al. 2007; Deloye et al. 2008; Wang et al. 2009, 2013) and recycled millisecond pulsars with irradiated companions (see, e.g., Breton et al. 2013).

First, we note that there seems to be an increasing trend of the light curves semi-amplitude with wavelength (see Table 1), whereby the $B-V$ colour is larger at the inferior conjunction of the companion than at the superior conjunction. Although this trend is affected by relatively large uncertainties, we note that it is opposite of what was observed during the 2004 observations (D'Avanzo et al. 2009) and what was expected from an irradiated companion since the heated side generally produces larger modulation amplitudes in the bluer part of the spectrum.
Table 1. Results of the photometry of XTE J1814-338 compared with the results of the observational campaign carried out on 2004 (D'Avanzo et al. 2009).

\begin{tabular}{lcccc}
\hline \hline Filter & $\begin{array}{c}\text { Semi-amplitude } \\
(\mathrm{mag})\end{array}$ & $\begin{array}{c}\text { Mean } \\
\text { magnitude }\end{array}$ & $\begin{array}{c}\text { Maximum } \\
\text { (phase) }\end{array}$ & $A_{\lambda}$ \\
\hline \multicolumn{5}{c}{ 2004 data (D'Avanzo et al. 2009) } \\
\hline$V$ & $0.52 \pm 0.08$ & $23.29 \pm 0.04$ & $0.48 \pm 0.02$ & $0.87 \pm 0.03$ \\
$R$ & $0.32 \pm 0.08$ & $22.52 \pm 0.03$ & $0.43 \pm 0.02$ & $0.66 \pm 0.06$ \\
$I$ & $0.33 \pm 0.10$ & $21.79 \pm 0.04$ & $0.44 \pm 0.02$ & $0.50 \pm 0.03$ \\
\hline \multicolumn{5}{c}{ 2009 data (this paper) } \\
\hline$B$ & $0.52 \pm 0.10$ & $24.44 \pm 0.07$ & $0.44 \pm 0.02$ & $1.17 \pm 0.03$ \\
$V$ & $0.63 \pm 0.10$ & $23.75 \pm 0.08$ & $0.47 \pm 0.02$ & $0.87 \pm 0.03$ \\
$R$ & $0.66 \pm 0.09$ & $23.43 \pm 0.05$ & $0.56 \pm 0.07$ & $0.66 \pm 0.06$ \\
\hline
\end{tabular}

Notes. The magnitude values are not corrected for reddening, whose parameters are reported in the last column. The 2004 I band results are obtained in this paper (text for details). The errors are indicated with a $90 \%$ c.l.

A possible explanation for this puzzling behaviour is that some further component is contributing to the optical emission with the companion star. In particular, the presence of a residual accretion disc during the quiescent state could explain our results. Since the RXTE/All Sky Monitor light curve of XTE J1814-338 reveals no outburst between 2004 and 2009, it is possible that the accretion disc around the compact object grew during this period, thus increasing its relative contribution to the total quiescent optical emission of the system. The disc is expected to be a hot structure with an emission peak at lower wavelengths with respect to the companion star, which peaks around the $V$ band ( $\sim 500 \AA$ A, D'Avanzo et al. 2009). The $B$ and $V$ band light curves could be more perturbed for this reason by the presence of the disc than the $R$ band one. Unless there are disc features displaying orbital variability (e.g. an eclipsed hot spot), its contribution to the total flux is a constant value. As a result, the amplitude of the light curves is attenuated. This effect is expected to be more important in the bluer bands since the disc spectrum should peak at a higher temperature than the companion.

Furthermore, we obtain unabsorbed mean colours $(B-V)=$ $0.39 \pm 0.11 \mathrm{mag}$ and $(V-R)=0.11 \pm 0.12 \mathrm{mag}$ from our photometry (computed assuming $E(B-V)=0.29$; D'Avanzo et al. 2009). If we consider these values as the colours of a main sequence star (as the companion star of XTE J1814-338 probably is; Krauss et al. 2005; D'Avanzo et al. 2009), they translate into inconsistent surface temperatures (between $6250 \mathrm{~K}$ and $7000 \mathrm{~K}$ from $(B-V)$ and between $11400 \mathrm{~K}$ and $7300 \mathrm{~K}$ from $(V-R)$; Cox 2000), providing a further indication of a two-component emission.

\subsection{Comparison between 2004 and 2009}

We note that the system was significantly fainter in the optical in 2009 than in 2004. Comparing the two datasets (Table 1) we find that the mean $V$ and $R$ band luminosities are decreased by $0.46 \pm 0.08 \mathrm{mag}$ and $0.91 \pm 0.06 \mathrm{mag}$, respectively. The combined $V$ and $R$ band light curves obtained from both datasets and rescaled to the same mean magnitude are reported in Figs. 3 and 4 . While the combined $V$ band curve can be satisfactorly fitted with a single sinusoid plus constant model, this is clearly not possible with the $R$ band data (Fig. 4) because of a significant phase offset. 


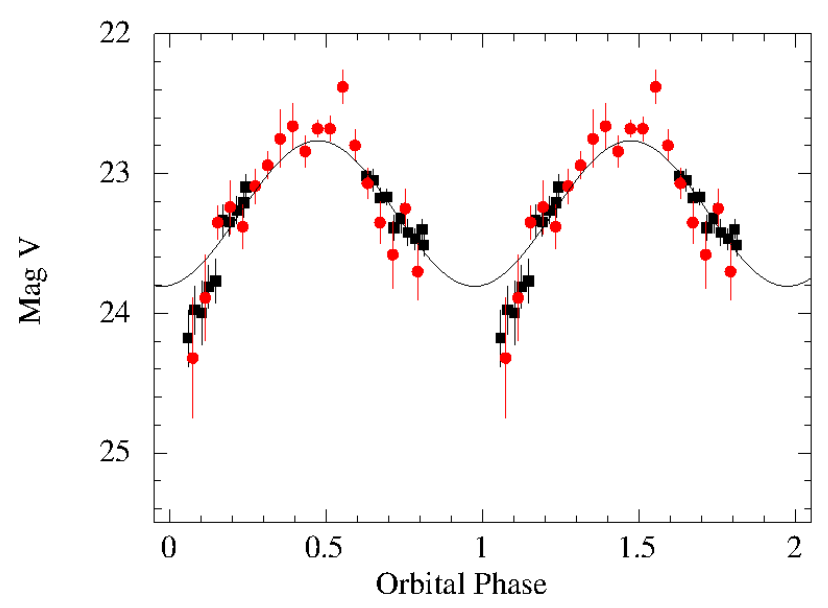

Fig. 3. $V$ light curve taken in 2009 (dots, this work) and the one taken in 2004 (squares, D'Avanzo et al. 2009). Both curves are normalized to the mean 2009 magnitude.

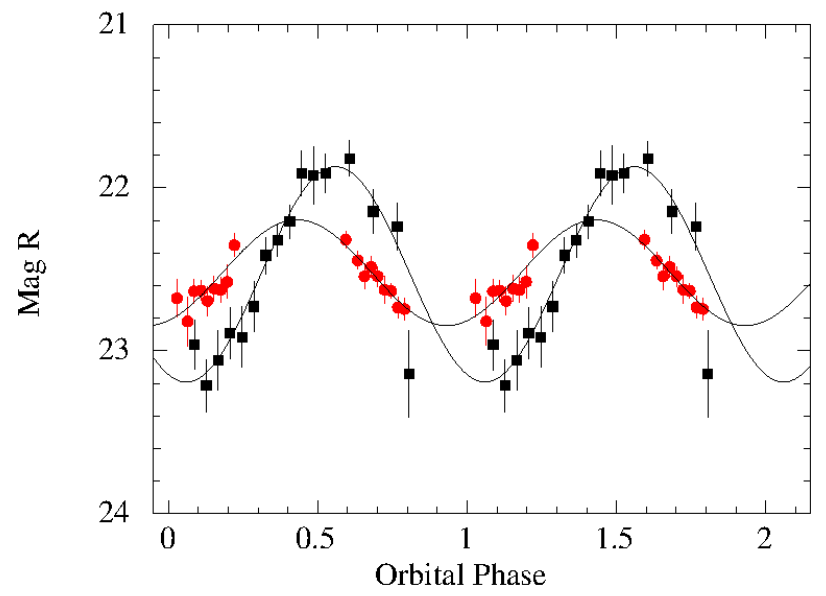

Fig. 4. $R$ light curves of D'Avanzo et al. (2009) (dots) and the one referred to the 2009 data (squares). Both curves are normalized to the mean 2009 mag.

This behaviour is unexpected if the irradiated star is responsible for the entire quiescent emission of the system. In this case we would expect to see all light curves in the same band to peak at the same phase, since all the light curves should peak at phase 0.5 (i.e. superior conjunction of the companion) without any significant offset in that scenario. This suggests that part of the modulation arises from another location, such as a disc asymmetry (e.g. a hot spot) whose periodicity is different from the orbital period of the companion. However, this scenario would require similar phase shifts to be observed in light curves from other bands as well, which is not the case (Fig. 4). On the other hand, alternative explanations, like superhumps, which are due to disc precession, are most likely expected to occur during outburst phases.

Offsets with respect to phase 0.5 are also observed in the $B$ and $I$ band light curve maxima. However, we have data covering only a single epoch for such bands (2004 for the $I$ band, 2009 for the $B$ band) which prevent us from making a comparison (as we did for the $V$ and $R$ band) and speculate on the possible physical origin of this offset.

\subsection{Fitting with ICARUS}

To model the XTE J1814-338 optical light curves with a selfconsistent physical approach, we have fitted the data using the Icarus $^{4}$ light curve modelling suite for irradiated binary companions (Breton et al. 2012). This model comprises several free parameters, which are:

- orbital inclination;

- distance modulus;

- extinction in $J$ band;

- day side companion temperature in 2004;

- day side companion temperature in 2009;

- disc flux in $V$ band in 2004;

- disc flux in $R$ band in 2004;

- disc flux in $V$ band in 2009;

- disc flux in $R$ band in 2009;

- disc flux in $B$ band in 2009 .

Given a mass for the pulsar, one can use the pulsar mass function derived from the X-ray timing to determine the companion mass. Then, the back side temperature of the companion is determined using a mass-temperature relationship for low-mass brown dwarfs (Deloye et al. 2008).

We have performed the light curve fitting using a Markov chain Monte Carlo (MCMC) procedure similar to that reported in Wang et al. (2013). We use a Gaussian prior on the distance of $8.0\left(M_{\mathrm{ns}} / 1.4 M_{\odot}\right)$ with $20 \%$ uncertainty, which corresponds to the value derived from type I bursts by Strohmayer et al. (2003). For the reddening, we use $A_{J}=0.253 \pm 0.078$, which is the value reported in D'Avanzo et al. (2009) with conservative uncertainties inflated by a factor of three.

Assuming a neutron star mass of $1.4 M_{\odot}$, the code provides a satisfactory fit (Fig. 5) with a distance modulus of 15.2 (which corresponds to a system distance of $\sim 11 \mathrm{kpc}$ within $2 \sigma$ from the estimated distance from type I bursts), a mean companion day side temperature of $\sim 5500 \mathrm{~K}$, and a companion mass of $(0.23 \pm 0.06) M_{\odot}$ (in agreement with the estimates reported in D'Avanzo et al. 2009). We do not find any evidence of a variation of the day side temperature between 2004 and 2009. However, a clear change in the disc flux is found during this period with a decrease by a factor of 1.6 and 5 in the $V$ and $R$ band, respectively. This shows that the disc became fainter and bluer. The orbital inclination, on the other hand, is basically unconstrained. Finally, we note that similar results are found assuming a neutron star mass of $2.0 M_{\odot}$.

\section{Discussion}

A comparison and joint analysis of multi-band optical data of XTE J1814-338 over five years shows that the quiescent optical emission of the system is likely due to the combination of at least two different components, namely an irradiated companion star and a disc.

\subsection{The companion star}

The combined fit of the optical light curves (Sect. 3.4) yields a companion star mass of $(0.23 \pm 0.06) M_{\odot}$, which is a value typical of main-sequence $M$-type stars. On the other hand, the inferred companion day-side surface temperature of $\sim 5500 \mathrm{~K}$ (found to be constant during both the 2004 and 2009 obbservations) would imply an earlier spectral type (G-K) for a mainsequence star. This apparent discrepancy was already pointed out

4 Freely available at https://github.com/bretonr/Icarus 


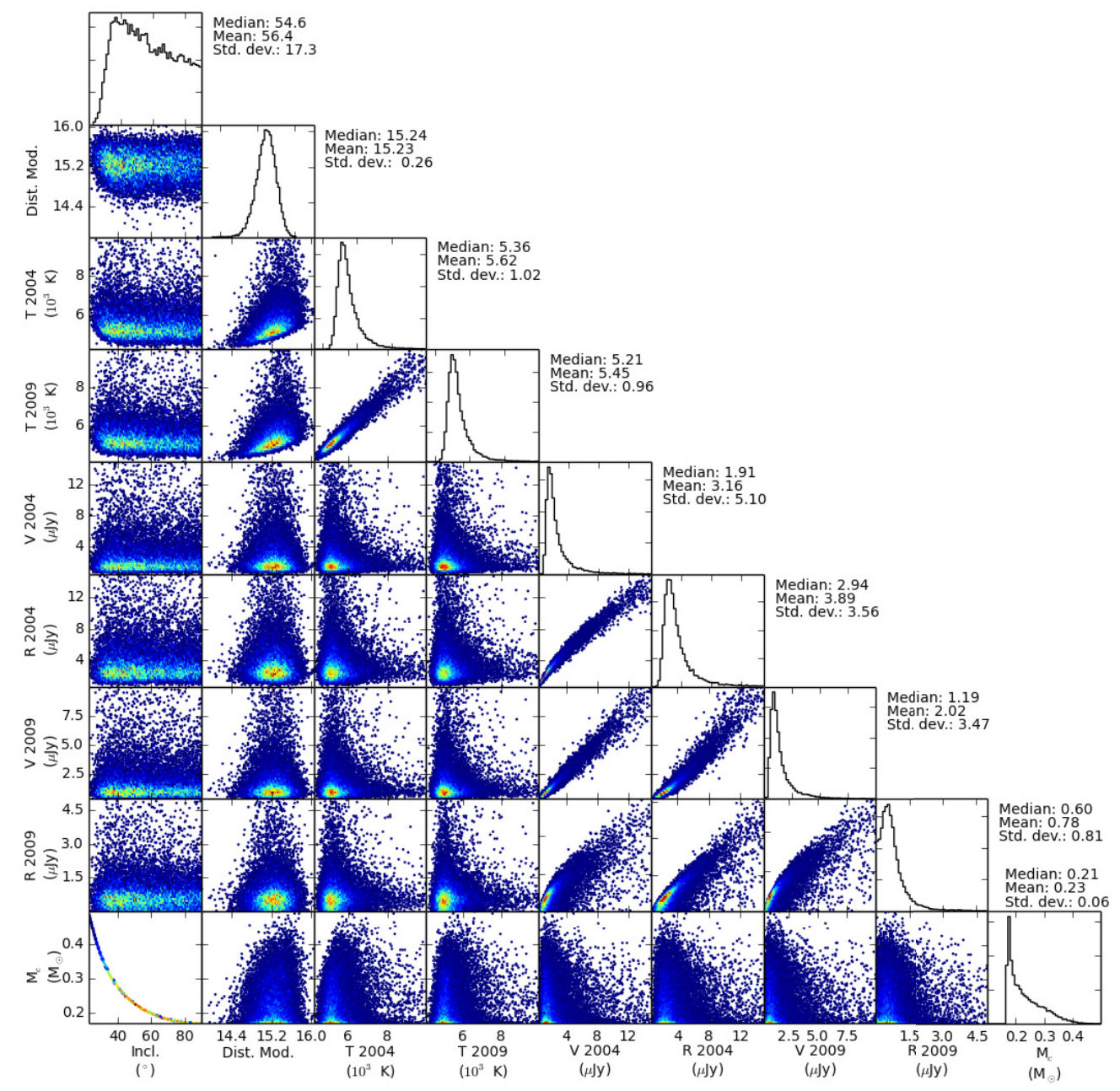

Fig. 5. One- and two-dimensional posterior distributions of key parameters obtained from our light curve fitting. The $R$ and $V$ denote the disc fluxes ( $\mu \mathrm{Jy}$ ) in their respective bands in 2004 and 2009; $T$ refers to the day side temperatures; $M_{\text {comp }}$ is the companion star mass. For these fits, we adopted $M_{\mathrm{ns}}=1.4 M_{\odot}$.

and discussed in D'Avanzo et al. (2009) as indirect evidence of a companion star that is heated by the relativistic particle wind of the neutron stars, and re-activated as a millisecond radio pulsar during the system quiescence. These results agrees with the "recycling scenario", in which neutron stars from AMXP systems switch between the state of accretion-powered pulsar to rotation-powered pulsar when the accretion stops (Alpar et al. 1982; Stella et al. 1994; Campana et al. 1998; Burderi et al. 2003; Patruno \& Watts 2012). Similar evidence was found by Wang et al. (2013) from the analysis of time-resolved optical imaging of SAX J1808.4-3658, the prototypical AMXP, which is collected during outburst and quiescence.

The absence of significant day-side temperature variation in the XTE J1814-338 companion during the five-year period suggests that the neutron star was already back to its rotationpowered state (see also Papitto et al. 2013) a few months after the end of the 2003 outburst. Assuming that irradiation simply acts to increase the companion's surface temperature, one can write the irradiation luminosity as $L_{\mathrm{irr}}=\sigma_{\mathrm{SB}}\left(T_{\text {day }}^{4}-T_{\text {night }}^{4}\right)$, where $\sigma_{\mathrm{SB}}$ is the Stefan-Boltzmann constant. If the irradiating flux is provided by the re-activated millisecond pulsar through its relativistic wind, one can relate the irradiation luminosity to the spin-down energy as $L_{\mathrm{sd}}=4 \pi a^{2} L_{\mathrm{irr}} / \epsilon_{\mathrm{irr}}$. Here, $a$ is the orbital separation and $\epsilon_{\text {irr }}$ is the irradiation efficiency, which can be ascribed to the albedo of the companion and the isotropy of the spin-down energy transported by the wind. Breton et al. (2013) showed that $\epsilon_{\text {irr }}$ for typical irradiated pulsar systems is in the range $0.1-0.3$. Hence, using the fitted temperature of the companion ( $~ 5500$ and $3300 \mathrm{~K}$ on the day and night side, respectively), we estimate the spin-down energy of the neutron star to be in the range $L_{\mathrm{sd}} \sim[6-17] \times 10^{34} \mathrm{erg} \mathrm{s}^{-1}$, which is typical of a 3.2 ms pulsar.

\subsection{The accretion disc}

While the companion star contribution to the overall quiescent optical emission remained constant, the disc component had displayed a significant change during the five years of our observations in terms of both flux and colour. In particular, the observed spectrum became bluer as the flux decreased from 2004 to 2009. A possible explanation of such long-term evolution is discussed in the following section.

\section{3. $A$ jet as a possible third component}

The system XTE J1814-338 is one of the three AMXPs (with SAX J1808.4-3658 and XTE J0929-314) for which a flux excess has been observed in the near-IR with respect to the 
A\&A 559, A42 (2013)

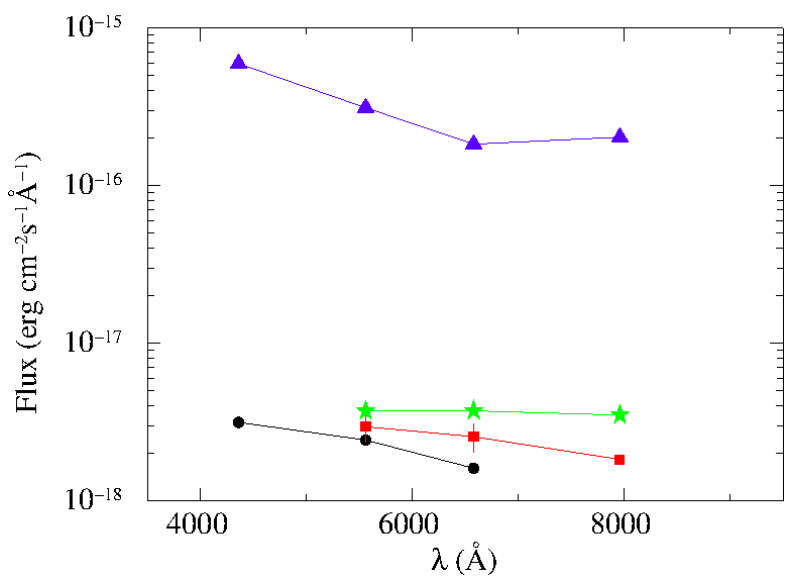

Fig. 6. Spectral energy distribution of XTE J1814-338 at four different epochs: 2003 (triangles, Krauss et al. 2005), 2004 (stars, D' Avanzo et al. 2009), 2007 (squares, D’ Avanzo et al. 2009) and 2009 (dots, this paper).

extrapolation of the UV-optical flux. Such excess has been observed for all three systems during the outburst phase with a wavelength corresponding to the $I$ band and has been interpreted as the contribution of a synchrotron emitting region, such as a synchroton jet (Krauss et al. 2005; Wang et al. 2001; Giles et al. 2005; Russell et al. 2007). In Fig. 6, we plot the evolution of the optical spectral energy distribution (SED) of XTE J1814-338 from the 2003 outburst to quiescence. The data plotted in Fig. 6 are taken from Krauss et al. (2005) and from Table 1 of this paper. We also added the corresponding VRI dereddened fluxes, which are derived from the low-resolution optical spectrum of XTE J1814-338 that were obtained in 2007 during quiescence (D'Avanzo et al. 2009). Despite the dramatic difference in terms of fluxes, the $(R-I)$ colour is almost constant in the June 2003 and May 2004 SED (Krauss et al. 2005 found $0.85 \pm 0.06$ and D'Avanzo et al. 2009, $0.73 \pm 0.05)$, suggesting that the extra component responsible of the $I$ band excess, as observed by Krauss et al. (2005) during the outburst, might still be present at the beginning of the quiescent phase. This component seems to be absent (or significantly fainter) in the following SEDs that are obtained in 2007 and 2009 during quiescence. The presence of a transient near-IR component due to synchrotron emission may provide an explanation to the large colour change observed in the quiescent optical SEDs. Indeed, we observe the spectrum becoming fainter and bluer. This could be explained if the jet component is progressively fading or its break frequency is moving towards longer wavelengths (i.e., lowering its contribution to the optical emission). Although jet emission in X-ray binaries is typically observed during active phases (Fender 2001; Russell et al. 2013), we note that it has recently been suggested to contribute to the quiescent optical emission from the black hole candidate Swift J1357.2-0933 (Shahbaz et al. 2013). However we are missing observations in the mid-IR and radio bands, where the possible jet contribution is expected to dominate (Fender 2001), to corroborate our claim.

An alternative explanation for the observed spectral evolution might be that the disc underwent a mass-transfer instability episode between 2004 and 2009 during which material from the outer part of the disc was transported inwards as a result of a viscous change. This effect could induce a fading of the outer disc region, whose emission is mostly driven by irradiation from the compact object $\left(T_{\text {irr }} \propto R^{-0.5}\right.$ whereas $T_{\text {visc }} \propto R^{-0.75}$, Hynes 2010). A similar explanation was proposed by Giles et al. (2005) to explain the spectral evolution observed during the outburst of the AMXP XTE J0929-314.

\subsection{The phase offset}

The main contribution to the quiescent optical emission observed in the 2009 data comes from the companion star, which is irradiated by the neutron star. Considering a companion star with a mass of $0.23 M_{\odot}$ filling its Roche lobe, we find that the whole star extends to $\sim 0.07$, which is consistent with the 0.06 observed phase offset.

Since the irradiation from the compact object should be symmetric, we hypothesize the presence of an additional component to the irradiation, which is likely a hot spot located in the accretion disc. Indeed, a consequence of the "hot spot irradiation" is that the inner face of the companion star would be be asymmetrically illuminated, causing the light curve maximum to shift from phase $\sim 0.5$ (as expected in the case of irradiation from the compact object) to phase $\sim 0.6$ (see Fig. 6 of Beuermann \& Thomas 1990). We note that evidence of asymmetrically irradiated companion stars caused by hot spot irradiation during quiescence in LMXB systems and cataclysmic variables have already been found (D’Avanzo et al. 2006; Beuermann \& Thomas 1990).

\section{Conclusions}

In this work, we presented the results of a phase-resolved multiband study of the quiescent optical counterpart of the AMXP system XTE J1814-338 that was obtained in 2009. Similar observations of the same source had been conducted by our team five years before at an earlier stage of the quiescent phase. The source experienced no outburst during this time period. The main results we obtained are summarized below.

- We have a clear detection of the quiescent optical counterpart of the system. The multi-band $(B, V, R)$ phase-resolved light curves show variability modulated at the $4.3 \mathrm{~h}$ orbital period.

- There is evidence of a two-component emission both in the 2004 and the 2009 observations, namely an accretion disc and a heated companion star.

- MCMC fitting of the multiband light curves enabled us to constrain some system parameters, such as the distance, the companion star's mass and surface temperature.

- Comparing the 2004 and 2009 datasets, we find that the system is fainter and bluer on average in the optical during the latter period. This is linked to the presence of the disc emission component, which has changed during the fiveyear period, while the companion contribution has remained constant. A suitable interpretation of the observed flux and spectrum is the presence of a third component in the 2004 emission, such as a jet, which is supported by the $I$ band excess that is observed for the system during the outburst in 2003 by Krauss et al. (2005). This component would have disappeared or severely weakened from 2004 to 2009, thus explaining the behaviour of the continuum component of the optical emission. Alternatively, a mass transfer instability due to viscous forces could explain the observed colour evolution.

- A phase offset is observed between the 2004 and 2009 $R$ band light curves (but not in the corresponding $V$ band data), suggesting that the companion star might be asymmetrically irradiated. We speculate that a combination of heating effects on the companion star from both the compact object and a hot spot in the disc could explain the observed phase offset. 
Acknowledgements. M.C.B. acknowledges Prof. Giuseppe Lodato (Università degli Studi di Milano) for supportive discussions and the INAF-Osservatorio Astronomico di Brera for kind hospitality during her bachelor thesis. S.C. and P.D.A. acknowledge the Italian Space Agency (ASI) for financial support through the ASI-INAF contract I/004/11/1. T.M.D. acknowledges funding via an EU Marie Curie Intra-European Fellowship under contract No. 2011-301355. R.P.B. was funded by a European Research Council Advanced Grant 267697 "4 Pi Sky: Extreme Astrophysics with Revolutionary Radio Telescopes".

\section{References}

Alpar, M. A., Cheng, A. F., Ruderman, M. A., \& Shaham, J. 1982, Nature, 300, 728

Archibald, A. M., Stairs, I. H., Ransom, S. M., et al. 2009, Science, 324, 1411

Beuermann, K., \& Thomas, H.-C. 1990, A\&A, 230, 326

Breton, R. P., Rappaport, S. A., van Kerkwijk, M. H., \& Carter, J. A. 2012, ApJ, 748,115

Breton, R. P., van Kerkwijk, M. H., Roberts, M. S. E., et al. 2013, ApJ, 769, 108

Burderi, L., Di Salvo, T., D’Antona, F., Robba, N. R., \& Testa, V. 2003, A\&A, 404, L43

Campana, S., Stella, L., Mereghetti, S., et al. 1998, ApJ, 499, L65

Campana, S., D’Avanzo, P., Casares, J., et al. 2004, ApJ, 614, L49

Cox, A. N. 2000, Allen's astrophysical quantities (Springer)

D’Avanzo, P., Muñoz-Darias, T., Casares, J., Martínez-Pais, I. G., \& Campana, S. 2006, A\&A, 460, 257
D’Avanzo, P., Campana, S., Covino, S., et al. 2007, A\&A, 472, 881 D'Avanzo, P., Campana, S., Casares, J., et al. 2009, A\&A, 508, 297

Deloye, C. J., Heinke, C. O., Taam, R. E., \& Jonker, P. G. 2008, MNRAS, 391, 1619

Fender, R. P. 2001, MNRAS, 322, 31

Giles, A. B., Greenhill, J. G., Hill, K. M., \& Sanders, E. 2005, MNRAS, 361, 1180

Homer, L., Charles, P. A., Chakrabarty, D., \& van Zyl, L. 2001, MNRAS, 325, 1471

Hynes, R. I. 2010 [arXiv: 1010 . 5770]

Krauss, M. I., Wang, Z., Dullighan, A., et al. 2005, ApJ, 627, 910

Markwardt, C. B., \& Swank, J. H. 2003, ATel, 179, 1

Papitto, A., di Salvo, T., Burderi, L., et al. 2007, MNRAS, 375, 971

Papitto, A., Ferrigno, C., Bozzo, E., et al. 2013, Nature, 501, 517

Patruno, A., \& Watts, A. L. 2012 [arXiv: 1206.2727]

Russell, D. M., Fender, R. P., \& Jonker, P. G. 2007, MNRAS, 379, 1108

Russell, D. M., Gallo, E., \& Fender, R. P. 2013, MNRAS, 431, 405

Shahbaz, T., Russell, D. M., Zurita, C., et al. 2013, MNRAS, 434, 2696

Stella, L., Campana, S., Colpi, M., Mereghetti, S., \& Tavani, M. 1994, ApJ, 423, L47

Stetson, P. B. 2000, PASP, 112, 925

Strohmayer, T. E., \& Watts, A. L. 2005, ApJ, 632, L111

Strohmayer, T. E., Markwardt, C. B., Swank, J. H., \& in't Zand, J. 2003, ApJ, 596, L67

Wang, Z., Chakrabarty, D., Roche, P., et al. 2001, ApJ, 563, L61

Wang, Z., Bassa, C., Cumming, A., \& Kaspi, V. M. 2009, ApJ, 694, 1115

Wang, Z., Breton, R. P., Heinke, C. O., Deloye, C. J., \& Zhong, J. 2013, ApJ, 765,151 